\title{
Tratamiento farmacológico de la obesidad
}

\begin{abstract}
El manejo farmacológico de la obesidad es complementario al tratamiento tradicional, no lo reemplaza ni puede indicarse aislado a la dieta y actividad física.

Los candidatos a la farmacoterapia son los que tienen un índice de masa corporal (BMI) mayor de 30; o bien un BMI mayor de 27 y la presencia de uno o más factores de riesgo cardiovasculares o apneas del sueño.

Se considera que un descenso de $2 \mathrm{~kg}$ o más durante el primer mes o bien una caída mayor al $5 \%$ del peso inicial a lo largo de seis meses son resultados efectivos.

Las únicas drogas que demostraron beneficios en el descenso de peso a mediano plazo son la sibutramina y el orlistat. Hasta la fecha no hay trabajos que hayan evaluado el efecto a largo plazo del tratamiento con drogas sobre los eventos y/o mortalidad cardiovascular.
\end{abstract}

\section{Introducción}

La obesidad es una de las enfermedades crónicas que más ha aumentado su incidencia en las últimas décadas, especialmente en los países industrializados. Su alta prevalencia, las diferentes condiciones de morbilidad que provoca y las complicaciones a largo plazo hacen que el médico de atención primaria se encuentre obligado a comprender y abordar este problema.

\section{Pronóstico}

Lamentablemente, los resultados de las distintas estrategias para el manejo de la obesidad son, en general, desalentadores. La mayoría de los obesos no adhiere a los tratamientos, probablemente porque se requiere mucha voluntad para cambiar hábitos que están fuertemente incorporados en la personalidad del paciente. Teniendo en cuenta que la obesidad es una condición crónica, lamentablemente, resulta muy difícil de sostener la conducta a lo largo del tiempo para que el beneficio sea prolongado. Más de la mitad de los pacientes que se encuentra bajo tratamiento sufre fuertes cambios emocionales, ansiedad y depresión. Por otro lado, sin tratamiento la enfermedad progresa, repitiéndose el ciclo y empeorando la situación y el pronóstico.

\section{Opciones de tratamiento}

De las distintas opciones para el manejo de la obesidad, el consejo dietario, el ejercicio y la reeducación del paciente para lograr cambios de hábitos y disminución del peso son las alternativas de manejo más costo-efectivas en el largo plazo.

El tratamiento farmacológico para la obesidad aparece como una herramienta complementaria a la dieta, la actividad física y el cambio de hábitos. Su rol es muy cuestionado debido a su parcial eficacia ya que los tratamientos son largos, costosos y sólo mantienen sus resultados mientras el paciente los recibe.

Los candidatos potenciales a la farmacoterapia son los que tienen un índice de masa corporal (IMC, o BMI en inglés) mayor de 30; o bien un BMI mayor de 27y la presencia de uno o más factores de riesgo cardiovasculares o apneas del sueño.

\section{Objetivos del tratamiento}

El objetivo del tratamiento es alcanzar las metas de peso previamente acordadas entre el equipo terapéutico y el paciente, según sus posibilidades, condiciones y factores de riesgo.
Es importante incluir en la evaluación y el seguimiento los cambios en la tensión arterial, los valores de glucemia, el perfil lipídico, el BMI y el índice cintura-cadera (el perímetro de la cintura dividido por el de la cadera).

Se considera que un descenso de $2 \mathrm{~kg}$ o más durante el primer mes o una caída mayor al $5 \%$ del peso inicial a lo largo de seis meses son resultados efectivos.

En algunos trabajos se considera que una pérdida de peso de 10 a $15 \%$ es un resultado satisfactorio, considerándose excelente si supera el $15 \%$.

\section{Familias de drogas disponibles para el tratamiento de la obesidad}

Las drogas simpaticomiméticas se caracterizan por su efecto potencial de aumentar la presión arterial. Este grupo incluye a los bloqueantes de la recaptación de noradrenalina (mazindol) y de serotonina (sibutramina y fluoxetina); así como a los agonistas adrenérgicos directos (fenilpropanolamina) y los que estimulan la liberación de la noradrenalina como el dietilpropion, fentermina y fendimetrazine.

Los simpaticomiméticos reducen la ingesta estimulando la saciedad o disminuyendo el apetito. La sibutramina y el mazindol inhiben la termogénesis en animales y quizás también en seres humanos. Todas estas drogas se administran por vía oral y son rápidamente absorbidas. El pico plasmático es alcanzado en una o dos horas y la vida media plasmática es corta, excepto para la sibutramina y su metabolito activo. Todas son metabolizadas e inactivadas en el hígado.

Por otro lado están las drogas que alteran el metabolismo lipídico como el orlistat (inhibe a la lipasa pancreática en la luz intestinal) y los péptidos que actúan inhibiendo el apetito (ej. leptina, glucágon y neuropeptido-Y) que no están por el momento aprobados para su uso clínico por los organismos regulatorios de EE.UU (Food and Drugs Administration, FDA).

Por último, las drogas que actúan estimulando el consumo de energía como la efedrina y los agonistas de los receptores beta-3 adrenérgicos tampoco están aprobadas para el tratamiento de la obesidad.

Las drogas aprobadas por la FDA para un tratamiento "corto" de la obesidad (menos de 12 semanas) son: fentermina, dietilpropion, mazindol y fenilpropanolamina. Sin embargo, la obesidad es una enfermedad crónica y por lo tanto, para conseguir efectos significativos a largo plazo, suelen requerir tiempos más prolongados, siendo permanentes ("de por vida") las medidas no farmacológicas. 


\section{Evidencia que respalda las drogas aprobadas para el tratamiento de la obesidad}

\section{Fentermina}

Es estructuralmente similar a las anfetaminas pero con escaso o nulo efecto dopaminérgico, lo que disminuye el riesgo de abuso. Su eficacia fue demostrada en un único estudio de 36 semanas de duración donde se sometió a los pacientes al tratamiento continuo o intermitente con la droga, comparándolos con un grupo placebo en forma doble ciega y aleatorizada. Atodos los pacientes se les recomendó una dieta de $1000 \mathrm{kcal} /$ día más $30 \mathrm{mg}$ de la droga o placebo y un grupo de pacientes alternó cada cuatro semanas droga con placebo.

Comparados con los que recibieron placebo durante todo el período del estudio, los pacientes expuestos a la droga lograron una reducción de peso significativa que tambien se manifestó en los que la recibieron en forma intermitente. En este último grupo el descenso de peso fue más lento en los períodos libres de droga y se aceleró con la administración del fármaco. Si bien su frecuencia fue escasa, los que usaron la medicación tuvieron más efectos adversos (ej. insomnio $8 \%$ ) y solo un $3 \%$ del total abandonó el estudio por ellos. Una vez suspendida la medicación el descenso de peso se sostuvo entre seis y nueve meses ${ }^{1,2}$.

Los resultados de este ensayo fueron corroborados en varios estudios controlados de corta duración (hasta cinco meses). Sobre la base de estos resultados la fentermina se presenta como una droga segura, eficaz y bien tolerada. Sin embargo todavía no hay evidencia suficiente de su efectividad en el largo plazo.

La dosis usual es de $30 \mathrm{mg}$ por día y los efectos adversos más frecuentes son cefalea, insomnio e irritabilidad. No se aconseja en pacientes con hipertensión no controlada, glaucoma, depresión, hipertiroidismo y antecedentes cardiovasculares.

\section{Mazindo}

Si bien no existen trabajos controlados que avalen su uso, dos estudios observacionales mostraron una reducción significativa de peso en los pacientes que recibieron droga, así como un descenso de la presión arterial y de los niveles de colesterol, triglicéridos e insulina.

Se usa en dosis de $1 \mathrm{mg}$ tres veces por día y sus efectos adversos más frecuentes son insomnio, ansiedad, sudoración, xerostomía, náuseas y constipación. En un trabajo donde se sometió a 15 pacientes a mazindol durante 12 semanas se registraron severos efectos adversos cardíacos, en pacientes sin cardiopatía previa, tres episodios de fibrilación auricular y dos de síncope. Está contrraindicado en pacientes con cardiopatía ${ }^{3}$.

\section{Fenilpropanolamina}

Hay poca información sobre este fármaco. Los trabajos aleatorizados y controlados con placebo son muy cortos (seis a 14 semanas) y en pocos de ellos sus resultados expresan diferencias significativas ${ }^{4,5}$. Se usa en dosis de 20 a $75 \mathrm{mg}$ por día en una toma diaria y los efectos adversos más comunes son irritabilidad, ansiedad, temblor, cefalea y elevación de la presión arterial. Tiene las mismas contraindicaciones que la fentermina.

\section{Sibutramina}

La sibutramina es la única droga de las simpáticomiméticas que fue estudiada durante un año en varios estudios controlados y aleatorizados. Los resultados demostraron que asociada a una dieta de bajas calorías y actividad física es efectiva para bajar de peso en personas obesas adultas con o sin factores de riesgo coronario adicionales. El descenso de peso se sostiene mientras se usa la droga, pero el peso se recupera a los pocos meses de ser suspendida. Todos los trabajos que compararon el uso de sibutramina contra placebo evaluaron la cantidad de $\mathrm{kg}$ de peso disminuidos, su proporción con relación al peso basal, la cantidad de pacientes que lograba reducir el 5 o el $10 \%$ de su peso al final del período y la proporción de pacientes que lograron sostener el 25,50 y $100 \%$ del peso perdido al final del estudio. Todos los resultados fueron a favor de la sibutramina con relación al placebo y estadísticamente significativos ${ }^{6,7,8,9}$

Tambien existe evidencia de que la sibutramina mejora la distribución de la grasa corporal total (grasa visceral y corporal valorada tomográficamente) disminuyendo significativamente el tejido celular subcutáneo abdominal y la grasa visceral, principales indicadores de riesgo cardiovascular de la obesidad.

En pacientes obesos diabéticos, dislipémicos e hipertensos el uso de sibutramina mejora los valores de glucemia, hemoglobina glicosilada, triglicéridos, LDL, colesterol total y ácido úrico, aumentando los de HDL pero no en forma significativa. Aunque todavía no hay trabajos donde se haya planteado la valoración de estos resultados en forma primaria ni los efectos de la droga sobre ellos en forma independiente al descenso de peso, es probable que su uso sea favorable o que por lo menos no altere desfavorablemente el pronóstico.

La sibutramina se utiliza en dosis diarias de 10 a $15 \mathrm{mg}$ por la mañana monitorizando la tensión arterial y frecuencia cardíaca durante su uso. En caso de aumentar los valores se debe suspender. Está contraindicada en pacientes con HTAno controlada, enfermedad coronaria, arritmias, insuficiencia cardíaca, antecedentes de $\mathrm{ACV}$, insuficiencia hepática o renal severa y glaucoma de ángulo estrecho.

Los efectos adversos más frecuentes son sequedad bucal, anorexia, insomnio, constipación y cefalea, siendo en general muy bien tolerada.

No debe administrarse junto a otros inhibidores de la recaptación de serotonina, antitusivos ni descongestivos. No se ha evaluado su uso concomitante a ansiolíticos, antidepresivos ni anticonvulsivantes. Vale aclarar que la eritromicina y ketoconazol utilizan las mismas vías metabólicas.

\section{Fluoxetina}

La fluoxetina es un inhibidor de la recaptación de serotonina que actúa inhibiendo el apetito. No hay mucha evidencia a su favor. Un trabajo aleatorizado y controlado contra placebo de 20 semanas de seguimiento demostró disminución de peso significativa, pero al año todos los pacientes habían recuperado el peso. Se usa en dosis de $20 \mathrm{mg}$ por la mañana aumentando progresivamente hasta $60 \mathrm{mg} /$ día, siendo sus efectos adversos más frecuentes insomnio, diarrea, ansiedad e irritabilidad ${ }^{10}$.

\section{Orlistat}

El orlistat actúa inhibiendo a la lipasa pancreática evitando la hidrólisis de los lípidos ingeridos en la dieta y aumentando su eliminación fecal. En dos estudios aleatorizados y controlados se vio que asociando la droga con una dieta hipocalórica disminuye en forma significativa del 5 al $10 \%$ del peso corporal y hasta un $30 \%$ del tejido adiposo, efecto que se sostiene hasta un año luego de su suspensión. Paralelamente los pacientes disminuyeron los niveles de colesterol total, LDL, glucemia e insulina.

La dosis recomendada es de $360 \mathrm{mg}$ por día dividida en tres tomas con las comidas. El efecto adverso más común es la diarrea, las flatulencias, la urgencia y la incontinencia fecal, siendo con frecuencia causa de abandono terapéutico. 
En general las drogas administradas a los pacientes obesos no alteran su farmacocinética cuando se utilizan concomitantemente al orlistat, tampoco los anticoagulantes orales, anticonceptivos orales ni digoxina, reduciéndose la absorción de las vitaminas liposolubles E, D y beta-carotenos. El tratamiento es tres veces más caro que con sibutramina ${ }^{11,12}$.

\section{Conclusión}

El manejo farmacológico de la obesidad es complementario al tratamiento tradicional, no lo reemplaza ni puede indicarse en forma separada a las otras medidas.

La dieta, la actividad física y el cambio de hábitos continúan siendo la clave en el tratamiento.

Como la obesidad es una enfermedad crónica, el tratamiento farmacológico debería realizarse con medicación que pueda utilizarse a largo plazo y las únicas drogas que demostraron beneficios a mediano plazo son la sibutramina y el orlistat, siendo la primera probablemente más costo-efectiva.

De incorporar a las estrategias para el tratamiento de la obesidad una intervención farmacológica a largo plazo, la droga debería no solamente lograr un descenso significativo del peso, sino principalmente un descenso de la morbilidad y mortalidad que la obesidad genera. Hasta la fecha ningún trabajo se ha diseñado para evaluar estos resultados. Existen resultados "intermedios" con beneficios no significativos en el descenso de los niveles sanguíneos de colesterol, glucemia, LDL, triglicéridos, hemoglobina glicosilada y tensión arterial. Hasta la fecha no hay trabajos que hayan evaluado la relación de estas drogas con los eventos y la mortalidad cardiovascular, enfermedad que conlleva la principal morbimortalidad de los pacientes obesos. Esperemos poder resolver estas dudas en un futuro cercano.

\section{Resumen para Ilevar al consultorio}

El uso de drogas se debe pensar como un tratamiento que debe acompañar la dieta y la actividad física en pacientes seleccionados (BMI>30 $0>27$ con aumento de riesgo vascular).

Las únicas drogas que demostraron beneficios en el descenso de peso a mediano plazo son la sibutramina y el orlistat.

No hay evidencia acerca de su efectividad a largo plazo, tanto en el peso como en los eventos y/o mortalidad cardiovascular.

La magnitud del beneficio es en general pequeño y no se mantiene al suspender en tratamiento.

\section{Dr. Diego Terceiro [ Unidad de Medicina Familiar y Preventiva. Hospital Italiano de Buenos Aires ]}

\section{Referencias}

1.Munro JF, McCuish AC, Wilson EM et al. Comparison of continuous and intermittent anorectic therapy in obesity. BMJ. 1968;1:352-354. 2.Cerulli J, Lomaestro BM, Malone M. Update on the pharmacotherapy of obesity. Ann Pharmacother 1998;32:88-102.

3.Gary Glazer MD. Long-term Pharmacotherrapy of obesity. Arch Intern Med. 2001;161:1814-1824.

3.Gary Glazer MD. Long-term Pharmacotherrapy of obesity. Arch Intern Med. 2001;161:1814-1824.

4. Weintraub M, Ginsberg G, et al. Phenylpropanolamine versus placebo in combination with caloric restriction and physician-maneged behavior modification.
5.Greenway F. Adouble-blind clinical evaluation of the anorectic activity of pheylpropanolamine versus placebo. Clin Ther 1989;11:584-9. (abstract)

6.Mc Neally and Goa. Sibutramine. Areview of its contribution to the management of obesity. Drugs 1998. Dec;56(6):1093-1124.

7.Luque and Rey. Sibutramine: Aserotonine-norepinephrine reuptake-inhibitor for the treatment of obesity. Ann Pharmacoter 1999;33:968-978

8.Apfelbaum M et al. Long-term maintenence oof weight loss after a very-low calorie diet: efficacy and tolerability of sibutrrramine vs placebo. Ann J Med. 1999: 106: 179-84.

9. Terceiro D. Sibutramina: Una nueva droga para el manejo de la obesidad. Evidencia en Atención Primaria. 2001 May-Jun; Vol 4 NNo3: 83-84.

10. Goldstein DJ, rampey AH, et al. Fluoxetine: arandomized clinical trial in the treatment of obesity. Int J Obes Relat Metab Disord 1994;18:129-35. (abstract)

11. Aone-year trial to assess the value of orlistat in the management of obesity. Int J Relat Metab Disord 1997 Jun; 21 Suppl 3: S24-S30.

12. Randomised placebo-controlled trial of orlistat for weight loss and prevention of weight regain in obese patients. European Multicentre Orlistat Study Group. The Lancet 1998, July 18 ; 352: 167-72.

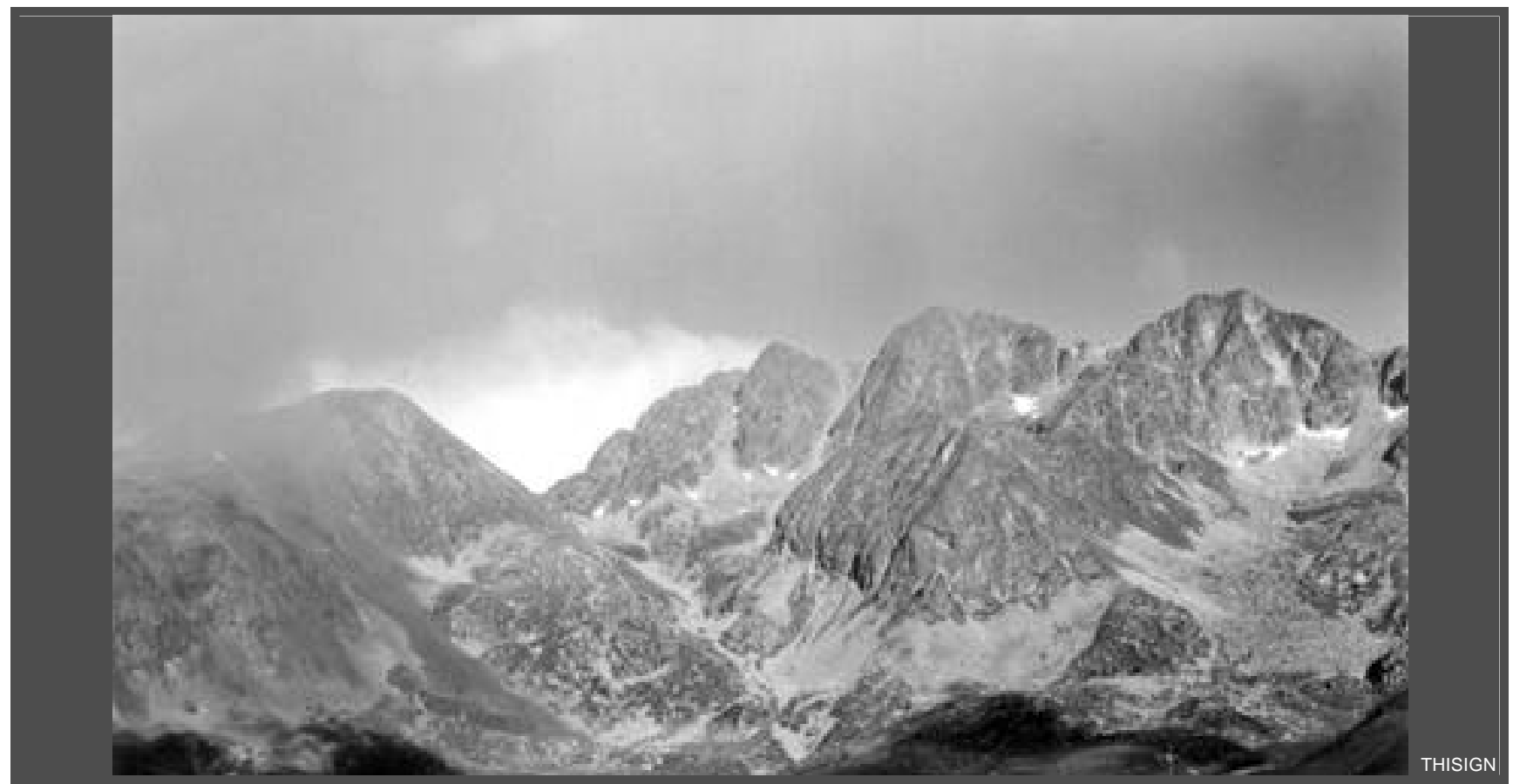

